\title{
TOPOLOGY DESIGN OF PLANAR RIGID-BODY MECHANISM USING CON- STRAINT FORCE DESIGN METHOD
}

\author{
J. C. Heo ${ }^{1}$, G. H. Yoon ${ }^{2}$ \\ ${ }^{1}$ Graduate student, School of Mechanical Engineering, Hanyang University, Republic of Ko- \\ rea
}

${ }^{2}$ Assistant Professor, School of Mechanical Engineering, Hanyang University, Republic of Korea (ghy@hanyang.ac.kr/gilho.yoon@gmail.com)

\begin{abstract}
This study develops a new design method called the constraint force design method, which allows topology optimization for planar rigid-body mechanisms. In conventional mechanism synthesis methods, it is possible to find out optimal locations of fixed number of joints and lengths of rigid-links efficiently. In order to circumvent the fixed topology limitation in designing optimal rigid-body mechanisms, the constraint force design method is presented [1]. Unlike other mechanism synthesis approaches, we perform kinetic analysis rather than kinematic analysis to find out optimal connectivity of rigid links as well as optimal locations of joints. The applicability and limitations of the newly developed design method are discussed in the context of its application to rigid-body synthesis problems.
\end{abstract}

Keywords: Topology optimization, Rigid-body mechanism, Constraint force design method.

\section{INTRODUCTION}

This research introduces the new synthesis method called constraint force design method for topology optimization of rigid-body mechanism [1]. For optimal synthesis design of rigid-body mechanism, various mechanism synthesis methods which consider path generation, function generation, and guidance for discovering optimal rigid-body mechanisms have been proposed [2-5]. As these synthesis methods optimize the dimensions of rigid links including the positions and types of joins, it is impossible to change topologies with these methods [2-5]. Despite the vast amount of research on analytical approaches, the synthesis of the optimal topologies of rigid-body mechanisms remain rare. In this study, we newly develop the constraint force design method for optimizing the topology of planar rigid-body mechanisms [1,6]. For optimization, we consider the two objective terms. The first objective term is the root mean square error between output path of synthesized rigid-body mechanism and optimal design of rigid-body mechanism. The second objective term is minimizing the number of links. For an optimization algorithm, the genetic algorithm with integer genotype encoding is employed [6]. 


\section{RIGID BODY MECHANISM ANALYSIS USING THE CONSTRAINT FORCE DE- SIGN METHOD}

In the constraint force design method, unit masses represent revolute or prismatic joints and the artificial forces maintaining the relative distances among the unit masses represent rigid links. For an example, let us consider the Figure. 1. To mimic the four-bar mechanism of Figure. 1(a), the length constraints are imposed among there nine masses during kinetic analysis in Figure. 1(b). Thus, for the present constraint force design method, it is possible to optimize the existences of the artificial forces.

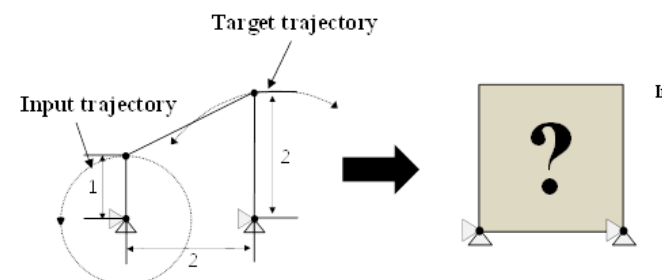

(a)

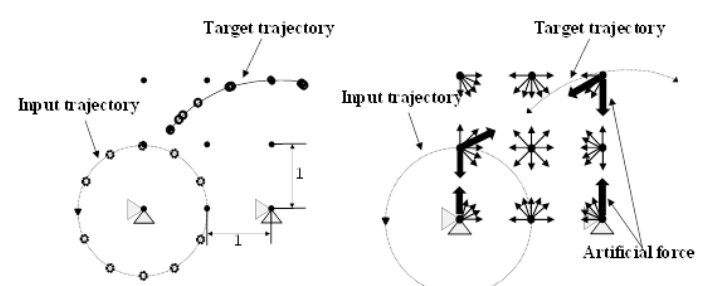

(b)

Figure 1. Constraint force design method. (a) The concept of the topology optimization for rigid-body mechanism design and (b) the constraint force design method.

\section{TOPOGOTY OPTIMIZATION FORMULATION FOR RIGID-BODY MECHA- NISMS}

\subsection{Parameterization of the existence of constraint forces}

For the topology optimization of rigid-body mechanisms, it is important to devise a proper parameterization method for the existence of length constraint force. It is found that we can assign one design variable to describe the existences of the artificial forces of the dynamic equations. (See [1] for more details). To implement this simple but effective feature numerically, we multiply the constraint force for the $k$-th link by the design variable $\gamma_{k}$

$$
\mathbf{r}=\gamma_{i} \mid \gamma_{i} \in[0,1] \text { and } i=1,2, \ldots, n l
$$

where $n l$ is the number of rigid-links. To illustrate concept of parameterization of the existence of constraint forces, we consider example in Figure 2. The design domain of the constraint force design method in Figure 2 contains four unit masses simulating revolute and prismatic joints and the six length constraints. To simulate rigid-body mechanism (Figure 2(b)), the parameterization of equation (1) is employed as shown in Figure 2(b). 


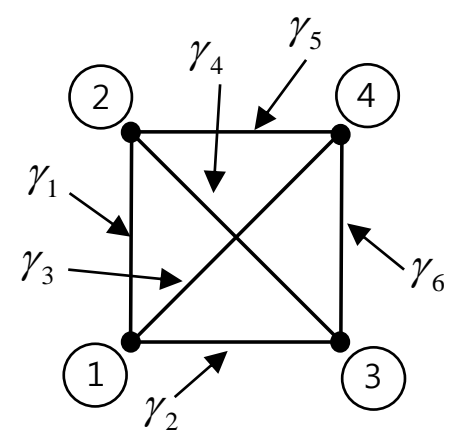

(a)

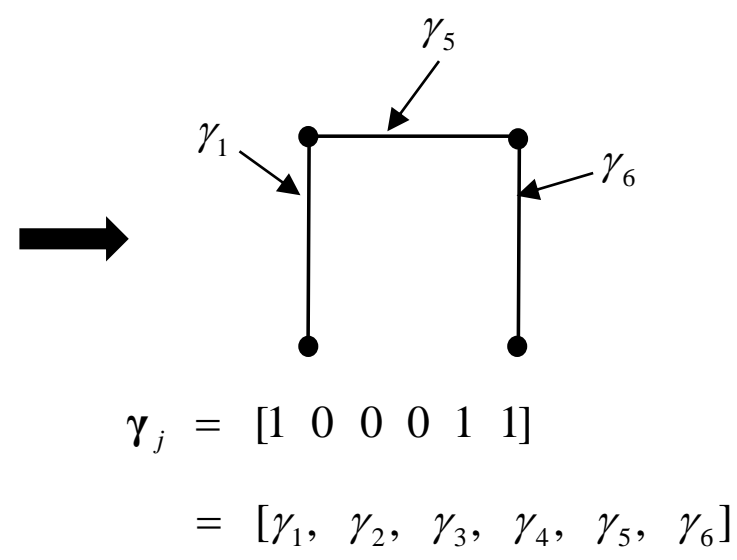

(b)

Figure 2. An illustrative example of the parameterization of the constraint force design mehtod.

\section{NUMERICAL EXAMPLE}

\subsection{Optimal synthesis of four-bar mechanism.}

To validate the performance of the constraint force design method, the numerical example of topology optimization of synthesis of planar rigid-body mechanism is considered in Figure 3. The design domain has nine unit masses simulating revolute of prismatic joints. The total 36 length constraints are considered for optimal synthesis of four-bar mechanism. By using the constraint force design method, the optimal four-bar mechanism of Figure 3(c) is found.

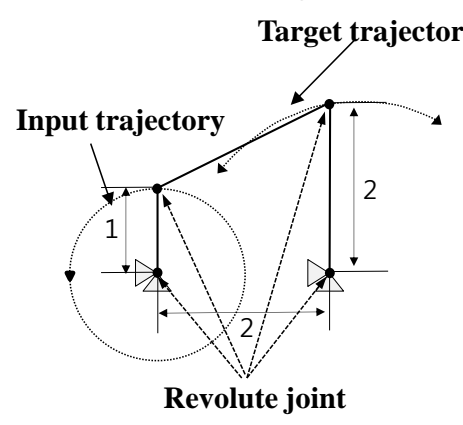

(a)

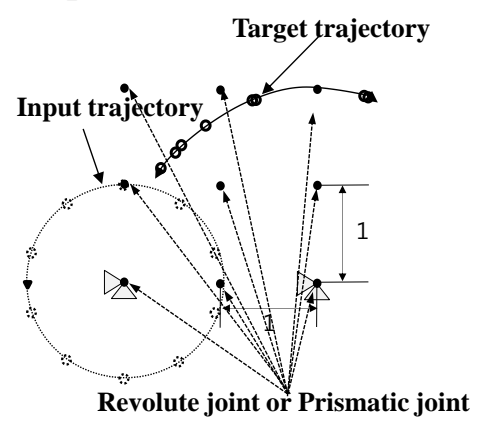

(b)

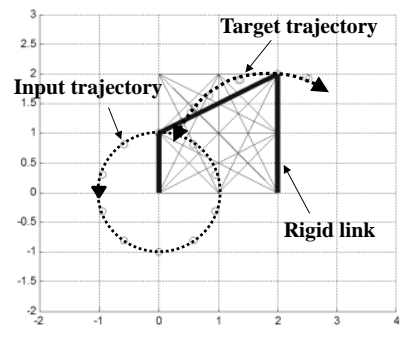

(c)

Figure 3. Numerical example of using constraint force design method. (a) The reference rigidbody mechanism, (b) the design domain of constraint force design method, and (c) the optimal result of rigid-body mechanism

\section{CONCLUSION}

In this paper, we introduce the constraint force design method for rigid-body mechanism synthesis method [1]. Unlike other synthesis method of rigid body mechanism, this newly developed method use kinetic analysis rather than kinematic analysis for design rigid-body mechanism. This synthesis method make possible to overcome several limitations of conventional 
synthesis method.

\section{REFERENCES}

[1] Yoon G.H. , Heo J.C. , Constraint force design method for topology optimization of planar rigid-body mechanisms, Computer Aided Design, 2012 in review.

[2] Mcgovern J. F., Sandor G. N., "Kinematic Synthesis of Adjustable Mechanisms .1. Function Generation”. J Eng Ind-T Asme. 95, 417-422, 1973.

[3] Wang Z. X., Yu H. Y., Tang J. S., "Study on rigid-body guidance synthesis of palanar linkage". Mech Mach Theory. 37, 673-684, 2002.

[4] Juinn C. S., Kota S., "Automated conceptual design of mechanisms". Mechanism and Machine Theory. 34, 467-495, 1999.

[5] Kawamoto A., Bendsøe M. P., Sigmund O., "Articulated mechanism design with a degree of freedom constraint". International Journal for Numerical Methods in Engineering. 61, 1520-1545, 2004.

[6] Holland J. H., “Genetic algorithms”. Scientific American. 1992. 